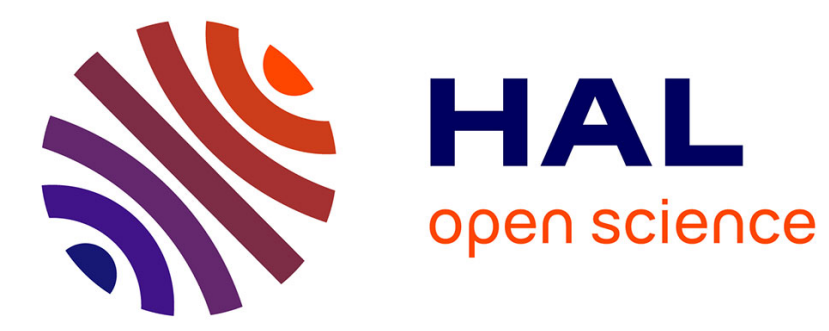

\title{
Questions on viral population biology to complete dengue puzzle
}

Christine Chevillon, Anna-Bella Failloux

\section{To cite this version:}

Christine Chevillon, Anna-Bella Failloux. Questions on viral population biology to complete dengue puzzle. Trends in Microbiology, 2003, 11 (9), pp.415 - 421. 10.1016/S0966-842X(03)00206-3 . pasteur-01713414

HAL Id: pasteur-01713414

https://hal-pasteur.archives-ouvertes.fr/pasteur-01713414

Submitted on 20 Feb 2018

HAL is a multi-disciplinary open access archive for the deposit and dissemination of scientific research documents, whether they are published or not. The documents may come from teaching and research institutions in France or abroad, or from public or private research centers.
L'archive ouverte pluridisciplinaire HAL, est destinée au dépôt et à la diffusion de documents scientifiques de niveau recherche, publiés ou non, émanant des établissements d'enseignement et de recherche français ou étrangers, des laboratoires publics ou privés. 


\title{
Questions on viral population biology to complete dengue puzzle
}

\author{
Christine Chevillon ${ }^{1}$ and Anna-Bella Failloux ${ }^{2}$ \\ ${ }^{1}$ Evolution des Systèmes Symbiotiques (CEPM, UMR CNRS-IRD 9926), 911 avenue Agropolis, BP 64501, \\ 34394 Montpellier Cedex 5, France \\ ${ }^{2}$ Ecologie des Systèmes Vectoriels, Institut Pasteur, 25 rue du Docteur Roux, 75724 Paris, France
}

\begin{abstract}
The mechanisms driving the current evolution of dengue remain unclear. We argue that disregarding the population biology of viruses has been a limiting factor, and present a hypothetical framework based on viral population biology. This allows us to pinpoint three flaws that need to be addressed: (1) a large underestimation of the viral polymorphism within populations; (2) a disregard of viral loci other than that encoding the antigenic envelope; and (3) the neglect of host-driven selection, especially that of vector-driven selection.
\end{abstract}

The aetiology and long-term history of dengue disease are well known. The pathogenic agents involved are viruses from four Flavivirus SISTER-SPECIES (see Glossary), each defining a distinct SEROTYPE: DEN-1, -2, -3 and DEN-4, respectively. These viruses have a single-strand RNA genome, in which $3^{\prime}$ and $5^{\prime}$ non-coding regions (NCRs) are found on either side of an open-reading frame encoding structural (C, M, E) and non-structural (NS1, NS2A, NS2B, NS3, NS4A, NS4B, NS5) proteins. All of these loci intervene in viral replication via complex virus-virus and virus-host interactions which are beginning to be identified in vitro [1,2]. DEN viruses are transmitted to humans by Aedes (Stegomyia) mosquitoes, with the two most frequent vector species being the peri-domestic and competitive invaders Ae. aegypti and Ae. albopictus. Phylogenetic studies based on NS5, E or $3^{\prime}$ NCR strongly support each other [3-6]: five to 10 centuries ago, sylvatic viruses of each DEN species were independently captured by humans, and since then, human-infecting viruses have diverged from sylvatic viruses. Therefore, the current evolution of dengue disease strictly depends on the evolution of the relationships between human-infecting viruses and their environment, i.e. their three main hostspecies (Homo sapiens, Ae. aegypti, and Ae. albopictus).

Dengue evolution is currently characterized by an overall emergence in the tropics, a continuous increase in morbidity and a tendency for a reduction in interepidemic periods [7]. Both environmental degradations and intensification of the traffic of humans and goods have greatly facilitated dengue emergence. Environmental degradations facilitate the multiplication of vector habitats in the vicinity of humans, thereby enhancing the opportunities of human-vector contact [8]. Traffic intensification promotes long-range dispersal of vectors [9] and viruses $[10-12]$. By contrast, the mechanisms regulating dengue morbidity and epidemics remain to be elucidated. In this article, we argue that the neglect of viral population biology partly explains the current problems with clarifying such mechanisms.

Problems related to dengue morbidity and epidemics Whatever the species involved, dengue disease displays various pathologies $[13,14]$, regrouped into three categories, ranking from silent infections to dengue fevers (DF) and dengue haemorrhagic fevers (DHF). This ranking order reflects both a gradual increase in morbidity and a gradual decrease in frequency. Indeed, silent infections are detected $a$ posteriori through the immunization pattern, whereas hospitalization is often required for DF cases and is obligatory for DHF cases. Silent infections are the most

\begin{abstract}
Glossary
ADAPTATION, CO-ADAPTATION, LOCAL ADAPTATION/MALADAPTATION: qualify the results of selection in terms of fitness increase. Co-adaptation and local adaptation are specific to host-pathogen systems where each species imposes selection on the other. Local adaptation of pathogens denotes that they have better fitness when infecting local rather than foreign hosts (local adaptation of hosts results in better host resistance to infection by local, rather than foreign, pathogens). Opposite patterns define local maladaptation.

Co-eVolution: denotes (1) that distinct species conjointly evolved for a while, such as dengue viruses with their hosts [3-6]; or (2) that two species are currently applying selection on each other, in the way that DEN viruses and their vector are considered to do. It is noteworthy that the first definition involves a longer time-scale and does not necessarily require selection: genetic drift between isolates captured by different host species would create divergence between them.

GENETIC DRIFT: chance process that occurs at each reduction in demographic size and induces random changes in polymorphism.

POLYMORPHISM: genetic variability observed at one locus.

SEROTYPE: a group of pathogens that elicit the same immune response. After a couple of months of cross-immunity protecting against all dengue viruses, a strict overlap of the four dengue serotypes and the four DEN species takes place.

SISTER-SPECIES: The four dengue serotypes (see definition above) define four sister-species of viruses, as (1) the corresponding viruses are genetically tightly related to one another, (2) recombination occurs within but not across serotypes and (3) any pair of viruses from the same serotype remains more closely related than any pair belonging to different serotypes.

VERTICAL/HORIZONTAL TRANSMISSION: Vertical transmission defines the transmission of a pathogen from parents to descendants (usually from mothers to offspring, as seems to be the case for dengue viruses in some populations of vectors). Horizontal transmission defines a pathogen transmission between unrelated individuals.

VIRULENCE: denotes here the part of morbidity that is determined by pathogen genetics.
\end{abstract}


frequent (representing up to $90 \%$ of the total burden in some areas and age groups [15]) and DHF are the rarest [7]. Therefore, there are two approaches to dengue epidemics. On the one hand, the immunization pattern of a population provides precise information on the number, identity and prevalence of the DEN species that were present [16-18]. On the other hand, because data on genetic polymorphism within DEN species are usually restricted to a few DF and DHF cases per population, the local viral diversity is largely underestimated.

Differences between DF and DHF cases have been studied with the aim of identifying the risk factors of dengue morbidity. Research has long focused exclusively on human characteristics and human-virus interactions. An important result was the finding that human genetics interferes with the susceptibility to develop DHF [7]. A more controversial issue is the 'antibody-dependent enhancement $(\mathrm{ADE})$ hypothesis'. This hypothesis analyses the infectious process from three different perspectives: biological, pathological and from the perspective of epidemiological dynamics. From a biological perspective, it is assumed that the viral load in secondary infections is enhanced by the interaction of dengue viruses with heterospecific antibodies [19]. This mechanism has faced so many critics that it now seems, at best, inconclusive [20]. From a pathological perspective, ADE predicts that the risk of developing DHF would be higher in secondary infections. The intensive epidemiological surveys listed in Table 1 do not strongly support this prediction in areas other than Thailand, and, even there, support is mixed with adverse evidence. Finally, from the perspective of epidemiological dynamics, this theory presumes that $\mathrm{ADE}$ would a priori create epidemic crises, preventing each DEN species from out-competing the others, and thereby facilitating the co-circulation of distinct species [21]. Conclusions are nevertheless unclear when this model is applied to a field survey (Table 1). Furthermore, this model treats dengue as a directly transmitted species [21], and its conclusions about the prevention of viral competition by $\mathrm{ADE}$ could not be supported when introducing a vector compartment because, contrary to humans, vectors never recover from infection [22].

Our understanding of the mechanisms that determine morbidity and epidemics is influenced by two contrasting hypotheses: one unsubstantiated, but predominant, null hypothesis $\left(\mathrm{H}_{\mathrm{O}}\right.$ : $\left.\mathrm{ADE}\right)$, and one that is corroborated by results $[7,10-12]$ but is disregarded $\left(\mathrm{H}_{1}\right.$ : variation in viral virulence within DEN species). It is worthy of note that, if

Table 1. Test of antibody-dependent enhancement (ADE) by intensive epidemiological data ${ }^{a}$

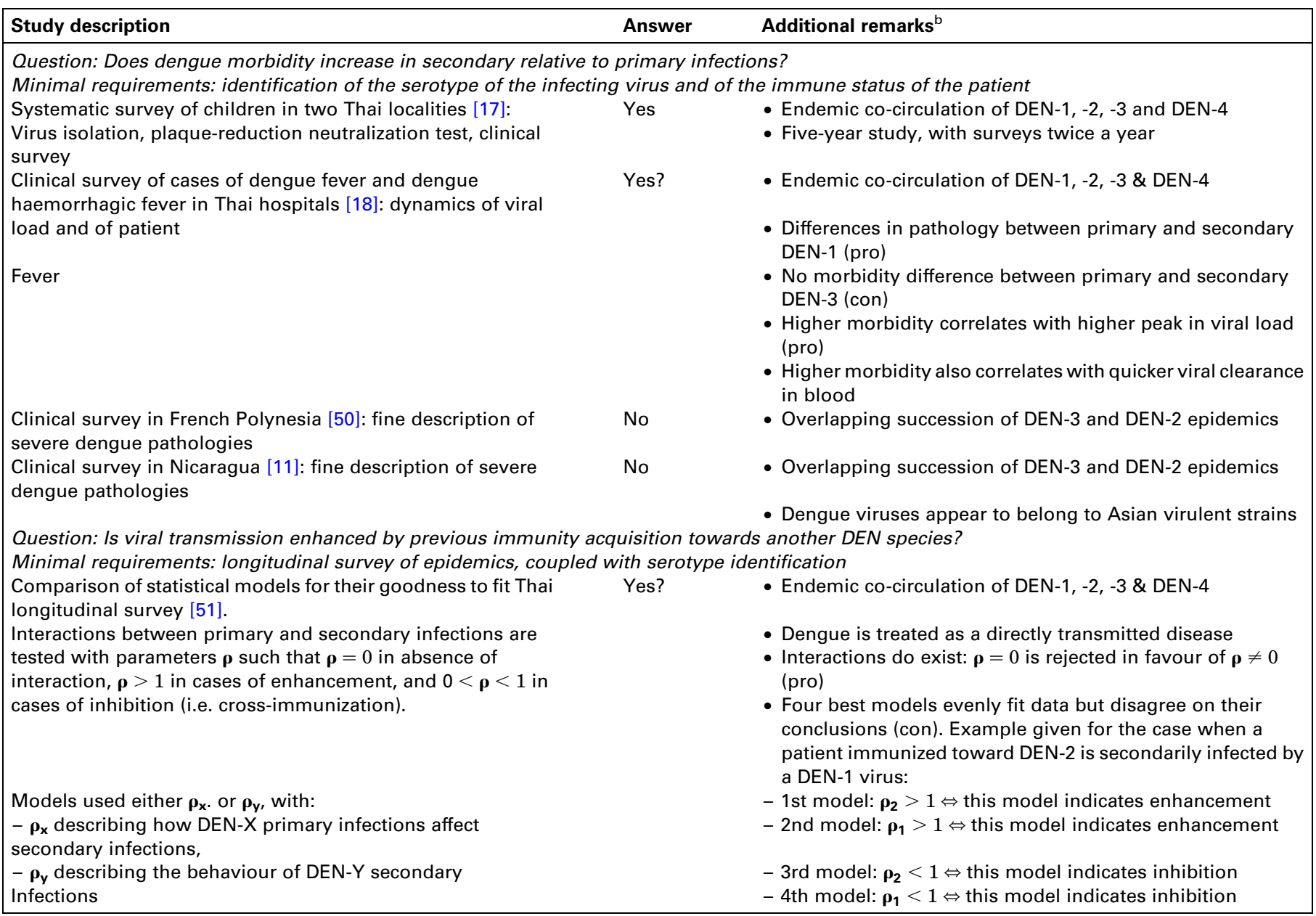

${ }^{a}$ The list of papers is not exhaustive but represents high-quality studies that document dengue epidemics with high morbidity in different areas, use complementary tools and were designed to test a component of ADE.

${ }^{b}$ The elements clearly arguing for and against the ADE hypothesis are followed by (pro) and (con), respectively. 
these hypotheses are not mutually exclusive, they drastically differ in their minimal requirements for identifying the mechanisms that determine morbidity and epidemics. Under $\mathrm{H}_{\mathrm{O}}$, identifying the DEN species circulating and the immune status of human populations are necessary and sufficient conditions. Under $\mathrm{H}_{1}$, identifying how the polymorphism of each co-circulating DEN species evolves would be an additional requirement.

\section{Viral evolution within populations}

A succession of evolutionary factors acts on viral polymorphism (Box 1). RNA viruses are characterized by the highest mutation rates known $\left(\sim 10^{-4}\right.$ per site and replication round), quick replication and large populations. All of these characteristics ensure the creation and maintenance of high genetic diversity within hosts [23]. Frequent recombination among viruses of the same DEN species [24-26] further enhances within-host diversity. Indeed, prediction of a high genetic diversity within the host has been confirmed for viruses infecting humans [27] and is beginning to be reaffirmed for those infecting mosquitoes [26]. This trait is important because of its connections to GENETIC DRIFT and selection. First, the higher the viral diversity within a host, the greater is the expected genetic change (due to genetic drift) between this original diversity and the subset that will be transferred to the next host. This could induce random changes within the viral diversity encountered among local hosts, with the extent of change being enhanced when the probability of infection success decreases (for example, when a high frequency of human immunization is reached locally). It is worthy of note that lineage extinction was actually documented within DEN-3 and -2 in Thailand [28,29], although it remains unclear whether selection or genetic drift was involved. Identifying the number of viruses transmitted and the distribution of viral polymorphism among local hosts is required to settle definitively the importance of genetic drift. Development of quantitative polymerase chain reaction [30] and sequencing tools should help this research.

Second, high within-host diversity promotes withinhost competition, and hence facilitates the selection for the viral genotypes that are most suited to the current host. How this will affect the local viral diversity depends on the selective differences among alternative host-species and on the balance in the number of viral generations infecting each one. Human immunization against a serotype affects the transmission of all genotypes of that serotype, and thus is unlikely to change genotype frequencies. By contrast, the vector can act as a selective agent, and thus can change the frequencies of viral genotypes. Indeed, the polymorphism of some vector genes controls: (1) vector resistance to viral infection [31]; (2) vector competence, i.e. whether infected vectors would ensure human infection in subsequent blood-feedings [31]; and (3) maternal-inheritance of viruses in vector offspring [32]. Therefore, resistance genotypes in vectors could select against some viral genotypes more than others. In addition, some vector genotypes may direct the transmission of some viral genotypes towards either the vertical or horizontal route. Finally, these vector loci are likely to be selected by viral polymorphism since infection is associated with costs to vector fitness, at least in laboratory conditions [33]. In other words, this vector-virus system fits the standards of host-parasite CO-EVOLUTION, where the characteristics of the reciprocal selection pressures determine the distribution of selected polymorphism among populations (see [34-36] and references therein). Our understanding will be improved by developing tools which are diagnostic of the vector and viral loci involved in such selection, analysing their polymorphism within and among populations with substantial sampling sizes per population $(n>20)$ and by investigating the nature of these selection pressures.

\section{Distribution of viral polymorphism among populations}

The high levels of mutation, recombination and drift experienced within populations lead to the expectation of immigrating viruses rapidly diverging from their ancestral population. Any viral locus can thus be expected to display a large genetic differentiation among populations within DEN species. Rapid in situ evolution of viral polymorphism was indeed observed whenever investigators looked for it [25]. In addition, despite a large underestimation as a result of single-gene studies and sample sizes being too small, and therefore not representative of the population, the genetic diversity within DEN species was reported to be both high and geographically structured $[3,4,12]$.

Vectors and humans also display population genetic structure [37,38]; one would therefore expect that hostdriven selection pressures combine with mutation and recombination to make dengue viruses adapt rapidly to local hosts. This prediction has never been tested directly. Indirect support for vector-virus co-ADAPTATION has nevertheless been reported [39]. In cases of co-adaptation between vectors and viruses, infection issues (i.e. success probability, relative proportion of VERTICAL TRANSMISSION and HORIZONTAL TRANSMISSION, viral load dynamics) would not be determined solely by either the viral or vector genome but would also depend on the interaction between the two genomes. Indeed, this expected pattern was precisely that reported for vector competence and viral load when crossing three Ae. aegypti strains and three DEN-2 strains in blood-meal infections [39]. Moreover, this pattern was also reported for the probability of vertical transmission [40]. In keeping with this, viral immigration into a new population was seen to affect dengue pathogenesis in humans $[11,14]$. The immigration of virulent Asian strains in Nicaragua was associated with an unexplained decrease in virulence and change in dengue pathogenesis [11], which may have been a consequence either of an incomplete strain identification (inaccurately merging two distinct genotypes into one) or of the viral adaptation to new hosts. A gradual change in dengue pathogenesis was also documented along the course of a DEN-2 epidemic in French Polynesia [14], an area where epidemics are caused by viral immigrants that out-compete resident viruses $[10,16]$, thereby indicating local MALADAPTATION of resident viruses there. Unfortunately, it is impossible to know whether this local 


\begin{abstract}
Box 1. Dengue biology
Dengue morbidity is not associated with any particular serotype on a worldwide scale $[13,19]$, although an association may arise on a local scale [18]. It is usually believed that dengue viruses are quickly cleared in humans (within $\sim 7$ days [18]). The uncertainty about the frequency of silent infections renders incomplete the documentation of instantaneous viral prevalence in humans, especially in endemic areas, where the population might have been immunized over a long period of time. A local prevalence of around $80 \%$ was nevertheless given in epidemic situations [52]. Viral prevalence in mosquitoes is even less well documented, as is the cost of infection to vector fitness [33]. This is unfortunate because only data on the variation in viral prevalence, in frequency of vertical transmission and in infection cost would allow accurate estimations to be made of the actual role of vectors in selecting viral polymorphism.
\end{abstract}

\section{The geographical distribution of these viruses is widespread and still expanding \\ The four serotypes have co-occurred in South-East Asia since the Second World War; an intriguing mosaic distribution is detected now- adays among nearby Thai villages [52]. Apparently, the four serotypes started to accumulate in a sustainable pattern in Latin America. Only DEN-1 and DEN-2 were reported in Africa, with the exception of rare appearances of DEN-3 in Mozambique and Somalia (see Ref. [53] for historical review). Three to four serotypes have circulated for the past 40 years in the Caribbean and Pacific islands plus Australia; in these locations, sporadic epidemics, sometimes including an overlap of two serotypes, appear to be the rule $[10,16]$.}

The evolutionary factors acting on viral polymorphism (Fig. la) Viral life history involves HORIZONTAL TRANSMISSION between host species and VERTICAL TRANSMISSION in vectors. Human immunization and vector resistance genes inhibit infection, and hence transmission. Infectious contacts between humans and vectors (in round brackets in
Fig. Ib) determine a drift effect and a change in host-driven selection. Mutation, recombination and the selection driven by within-host competition co-occur in all phases indicated by an asterisk in Fig. la. Note that several steps in both the horizontal and vertical routes of transmission are under vector genetic control, and that multiple infections are not rare (inter- and intra-serotypes, see [54] and [26], respectively).

\section{Within-population partition of viral polymorphism between} vector and human compartments (Fig. lb)

Differences in viral genotypes are represented by host colours. A viral population includes any transmissible viruses, whether they are present in human or vector compartments, and thus clearly excludes the viruses failing vector infection. In humans, the only prediction is that the viral determinants of dengue morbidity would be in higher frequency in the visible viral burden, corresponding to cases of dengue fever (DF) and dengue haemorrhagic fever (DHF), relative to silent infections. In vectors, the only other indication known is that competent vectors (whose genes allow viral horizontal transmission) vary in frequency among nearby populations $(<20 \mathrm{~km}$ apart in Polynesian islands [55] or Ho Chi Minh city, Vietnam, [56]). It would be crucial to address the dependence of such results on the viral genotypes and on vector-virus genetic interactions to ascertain the 'rules' of dengue epidemics in vector compartments. Indeed, vector resistance genes may select against some viral genotypes or randomly change genotype frequencies by genetic drift in places where competent vectors are rare (Fig. Ib, question i). Moreover, vector genetic control of transmission routes raises the opportunity for vectors to differentially select the viral genotypes that are either vertically or horizontally transmitted (Fig. Ib, question ii). Finally, it will be necessary to address the nature of the connections between human and vector compartments, i.e. between groups of viruses classified in terms of human pathological signs and those classified in terms of vector-virus co-evolution. maladaptation concerned only the surveyed host (namely, $H$. sapiens) or if it involved both humans and vectors.

The emerging possibility of a co-evolution of dengue viruses with both local vectors and humans raises a new set of questions that has not yet been addressed. For example, how does the adaptation to local vectors affect pathogenesis in humans? Conversely, how does a change in the viral determinant of human pathogenesis affect the viral adaptation to local vectors? Does local adaptation occur, and, if so, are viruses locally adapted to vectors and/or to humans? Does local adaptation interfere with the genetic basis of virulence? Addressing these questions requires a complete documentation of local epidemics, including details of dengue morbidity, viral polymorphism and vector-virus interactions and/or a full identification of host-driven selection.

\section{Host-driven selection}

In vitro experiments suggest both the occurrence of hostdriven selection and an antagonism between human- and vector-driven selection. It is well known that multiple passage in mosquitoes or in C6/36 mosquito cells attenuates the virulence of dengue viruses for mammalian cell cultures and vice versa [41]. However, very little is known about the targets of host-driven selection in the viral genome.

Direct investigation of the occurrence of host-driven selection was only undertaken for $3^{\prime} \mathrm{NCR}$, and, indeed,
3 NCR appears to be antagonistically selected by insect and mammalian cells [42]. This is significant because 3 NCR was suspected to be a viral determinant of DEN-2 morbidity ([43], but also see [4]). How would vector-driven selection interfere with human pathology? Protocols of experimental evolution addressed this question for two RNA viruses [44-47]: the vesicular stomatitis virus (VSV), a facultative vector-borne virus displaying a wide array of hosts and transmission patterns, and the Eastern equine encephalitis virus (EEEV), a mosquito-borne alphavirus. These experiments used three designs of transmission: (1) transmission from insect cells to insect cells (selection for insect specialists); (2) transmission from vertebrate cells to vertebrate cells (selection for vertebrate specialists); and (3) an alternative transmission in insect and vertebrate cells (selection for generalists). Studies in VSV and EEEV led to the same conclusions. The selection for insect specialists drastically reduced competitive skills in vertebrate cells. Comparatively, the selection for vertebrate specialists drastically reduced competitive skills in insect cells. However, the competitive skills of generalists remained as good as those of insect specialists in insect cells and those of vertebrate specialists in vertebrate cells. Moreover, experiments on EEEV showed that its adaptation to insect cells occurs along with both an increase in the success of infection of these cells and a reduced population growth within these cells [47]. Therefore, the adaptation of EEEV to vertical transmission in insect cells 
(a)

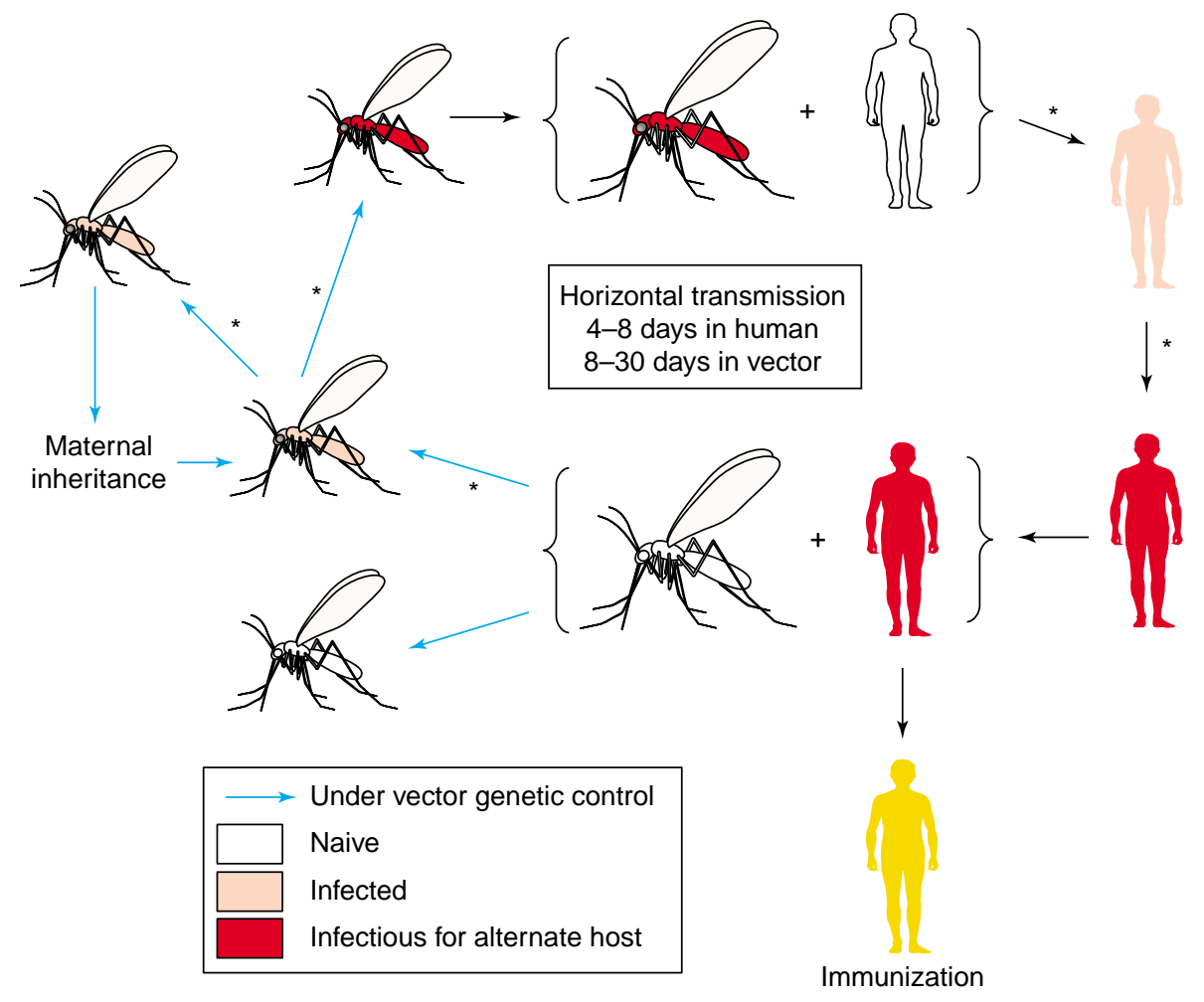

(b)

\section{Genotypes failing vector infection}

(i) Counter-selection and drift?

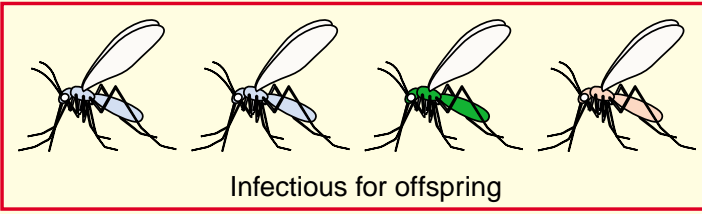

(ii) Differential selection?

$\downarrow$

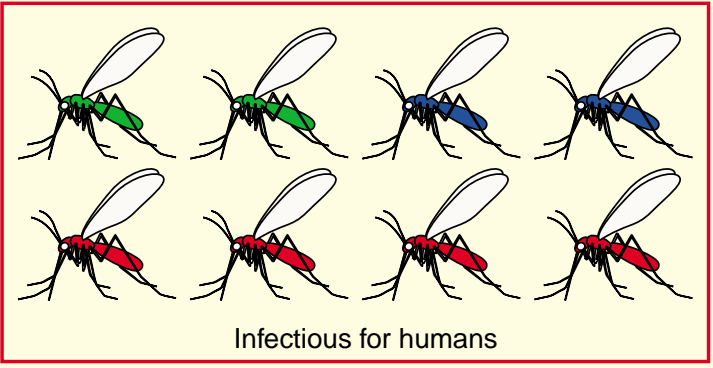

Global human burden

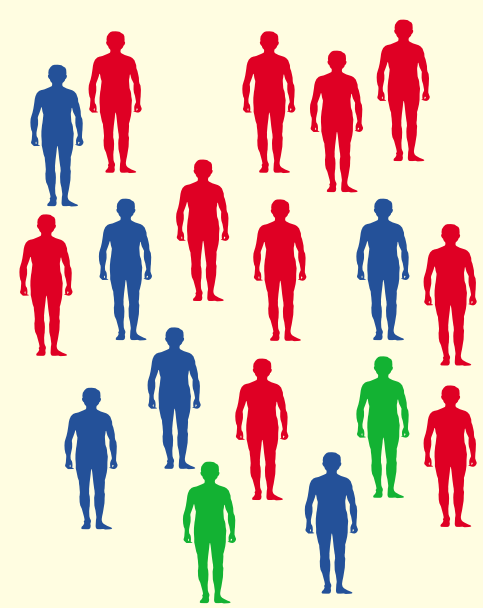

Visible fraction (DF and DHF)

TRENDS in Microbiology
Fig. I. Viral polymorphisms in humans and vectors.

reduced both its ability to compete in vertebrate cells and the probability of its being transmitted from insect to vertebrate cells. Investigating whether dengue viruses experienced similar trade-offs would require either the undertaking of similar experimental evolutionary protocols with these viruses, or the more complete characterization of the host-driven selection pressures and their targets in the genome of dengue viruses.
Looking at the most recent compilations of data [12,48], two factors have so far limited this characterization. First, viral genetics was mainly studied from isolates of human origin, thereby focusing on the output of human-driven selection. Second, viral polymorphism has rarely been studied at loci distinct from E. With theses biases, Twiddy et al. [12,48] concluded that a large predominance of functional constraints had strongly limited the divergence 
of viral polymorphism. However, whenever discrimination was possible, the conclusions differed between the subset of viruses that only experienced mosquito passage and the other viruses [48], confirming that mosquitoes select the viral polymorphism differently than do humans. More balanced sampling among alternative hosts in the field would be required to clarify the viral molecular targets of mosquito-driven and human-driven selection and to clearly address the population variation of both sources of selection. Moreover, population-focused data on viral prevalence in vector populations, infection costs for vector fitness, and on the frequency of vertical transmission are required to estimate correctly the actual role of vectors on viral polymorphism.

Finally, it is noteworthy that high morbidity can sometimes be deleterious for viral fitness. Vaughn et al. effectively reported in their longitudinal Thai survey that viral clearance in patient blood arose more rapidly, thereby reducing the period of transmission opportunity for vectors, in DHF cases relative to the cases of less severe diseases [18]. If this is confirmed in other populations, it would show that humans directly select for lower virulence. This point could be addressed by increasing the molecular characterization of the viruses co-circulating in endemic areas, using large sample sizes $(n>20)$, and/or by investigating the relationships, among local human isolates of dengue viruses, between morbidity in humans, infection ability of local vectors and the dynamics of viral load in these vectors.

\section{Conclusion}

Initially, the implicit assumptions about dengue characteristics were that they would obey universal rules, in which case transmission would be controlled by vectors, whereas pathogenesis and morbidity would be co-regulated by human history and viral genetics. We suggest that this hypothetical framework is likely to be invalid (see also Refs [6] and [20] for complementary arguments). We also suggest that an alternative hypothetical framework, based on viral population biology, looks promising. Although several studies have recently taken this direction [12,26,28,29], we have identified several questions that remain to be addressed and have listed possible ways to bring this about. The main 'takehome message' is the emphasis on two properties that should be taken into account by studies aimed at investigating the role of viral genetics in disease evolution. First, any viral gene is likely to evolve differently among populations. The observations of population variation in the selection experienced by viral genome $[12,48]$ and in viral evolutionary rate [49] have re-affirmed this prediction. It is crucial to stop underestimating the local levels of viral polymorphism; one way to do this would be to enlarge population samples.

Second, irrespective of the viral trait of interest, there is no reason to ignore vector-driven selection more than human-driven selection. Indeed, both hosts were shown to select the viral loci for which investigation had been undertaken ( $3^{\prime} \mathrm{NCR}$ and $\mathrm{E}$, see above). Moreover, there is no reason to target the $\mathrm{E}$ polymorphism more than that of other loci. Indeed, several viral loci appear to be putative determinants of morbidity, although overly restricted sample sizes prevent one from being able to trust these results fully ( $n=12$ for a worldwide survey of DEN-2; Ref. [43]). Collective effort on more appropriate sample sizes to increase the amount of molecular data, as well as viral sampling in vectors and silently infected patients, should help to improve our understanding of the population variation in dengue disease dynamics.

\section{Acknowledgements}

We are grateful to Thierry de Meeûs for his constant encouragement and helpful discussions. We also thank L. Rosen, F. Rodhain and M. VazeilleFalcoz for interesting feedback on earlier drafts, as well as the Editor and referees for useful criticism and editing comments.

\section{References}

1 Proutski, V. et al. (1999) Biological consequences of deletions within the $3^{\prime}$ - untranslated region of flaviviruses may be due to rearrangements of RNA secondary structure. Virus Res. 64, 107-123

2 Valle, R.P.C. and Falgout, B. (1998) Mutagenesis of the NS3 protease of dengue virus type 2. J. Virol. 72, 624-632

3 Wang, E. et al. (2000) Evolutionary relationships of endemic/epidemic and sylvatic dengue viruses. J. Virol. 74, 3227-3234

4 Shurtleff, A.C. et al. (2001) Genetic variation in the $3^{\prime}$ non-coding region of dengue viruses. Virology 281, 75-87

5 Gaunt, M.W. et al. (2001) Phylogenetic relationships of flaviviruses correlate with their epidemiology, disease association and biogeography. J. Gen. Virol. 82, 1867-1876

6 Holmes, E.C. and Twiddy, S.S. (2003) The origin, emergence and evolutionary genetics of dengue virus. Infect. Genet. Evol. 3, 19-28

7 Guzman, M.G. and Kouri, G. (2002) Dengue: an update. Lancet Infect. Dis. 2, 33-42

8 Rodhain, F. (1983) Maladies transmissibles par les Culicidés et urbanisation: un exemple de coévolution. Bulletin de l'Institut Pasteur $81,33-54$

9 Reiter, P. and Sprenger, D. (1987) The used tire trade: a mechanism for the worldwide dispersal of container breeding mosquitoes. J. Am. Mosq. Control Assoc. 3, 494-501

10 Chungue, E. et al. (1993) Molecular epidemiology of dengue 3 viruses and genetic relatedness among dengue 3 strains isolated from patients with mild or severe form of dengue fever in French Polynesia. J. Gen. Virol. 74, 2765-2770

11 Harris, E. et al. (2000) Clinical, epidemiologic, and virologic features of dengue in the 1998 epidemic in Nicaragua. Am. J. Trop. Med. Hyg. 63, $5-11$

12 Twiddy, S.S. et al. (2002) Phylogenetic relationships and differential selection pressures among genotypes of dengue-2 virus. Virology 298, 63-72

13 Kurane, I. and Ennis, F.A. (1997) Immunopathogenesis of dengue virus infections. In Dengue and Dengue Hemorrhagic Fever (Gubler, D.J. and Kuno, G., eds), pp. 273-290, CAB International

14 Deparis, X. et al. (1998) Changing clinical and biological manifestations of dengue during the dengue-2 epidemic in French Polynesia in 1996/97 - description and analysis in a prospective study. Trop. Med. Int. Health 3, 859-865

15 Guzman, M.G. et al. (2000) Epidemiologic studies on dengue in Santiago de Cuba, 1997. Am. J. Epidemiol. 152, 793-799

16 Deparis, X. et al. (1998) Possible dengue sequential infection: dengue spread in a neighbourhood during the 1996/97 dengue-2 epidemic in French Polynesia. Trop. Med. Int. Health 3, 866-871

17 Thien, S. et al. (1997) Risk factors in dengue shock syndrome. Am. J. Trop. Med. Hyg. 56, 566-572

18 Vaughn, D.W. et al. (2000) Dengue viremia titer, antibody response pattern, and virus serotype correlate with disease severity. J. Infect. Dis. 181, 2-9

19 Rothman, A.L. (1997) Viral pathogenesis of dengue infections. In Dengue and Dengue Hemorrhagic Fever (Gubler, D.J. and Kuno, G., eds), pp. 245-273, CAB International

20 Bielefeldt-Ohmann, H. (1997) Pathogenesis of dengue virus diseases: missing pieces in the jigsaw. Trends Microbiol. 5, 409-413

21 Ferguson, N. et al. (1999) The effect of antibody-dependent 
enhancement on the transmission dynamics and persistence of multiple-strain pathogens. Proc. Natl. Acad. Sci. U. S. A. 96, 790-794

22 Feng, Z. and Velasco-Hernandez, J.X. (1997) Competitive exclusion in a vector-host model of dengue fever. J. Math. Biol. 35, 523-544

23 Domingo, E. and Holland, J.J. (1997) RNA virus mutations and fitness for survival. Annu. Rev. Microbiol. 51, 151-178

24 Worobey, M. et al. (1999) Widespread intra-serotype recombination in natural populations of dengue virus. Proc. Natl. Acad. Sci. U. S. A. 96, $7352-7357$

25 Uzcategui, N.Y. et al. (2001) Molecular epidemiology of dengue type 2 virus in Venezuela: evidence for in situ virus evolution and recombination. J. Gen. Virol. 82, 2945-2953

26 Craig, S. (2003) Diverse dengue type 2 virus populations contain recombinant and both parental viruses in a single mosquito host. J. Virol. 77, 4463-4467

27 Wang, W.-K. et al. (2002) Dengue type 3 virus in plasma is a population of closely related genomes: quasispecies. J. Virol. 76, 4662-4665

28 Wittke, V. et al. (2002) Extinction and rapid emergence of strains of dengue 3 virus during an interepidemic period. Virology 301, 148-156

29 Sittisombut, N.A. et al. (2000) Possible occurrence of a genetic bottleneck in dengue viruses type 2 between 1980 and 1987 epidemic seasons in Bangkok, Thailand. Am. J. Trop. Med. Hyg. 57, 100-108

30 Laue, T. et al. (1999) Detection of dengue virus RNA in patients after primary or secondary dengue infection by using the TaqMan automated amplified system. J. Clin. Microbiol. 37, 2543-2547

31 Bosio, C.F. et al. (2000) Quantitative trait loci that control vector competence for dengue-2 virus in the mosquito Aedes aegypti. Genetics $156,687-698$

32 Mourya, D.T. et al. (2001) Horizontal and vertical transmission of dengue virus type 2 in highly and lowly susceptible strains of Aedes aegypti mosquitoes. Acta Virol. 45, 67-71

33 Joshi, V. et al. (2002) Persistence of dengue-3 virus through transovarial passage in successive generations of Aedes aegypti mosquitoes. Am. J. Trop. Med. Hyg. 67, 158-161

34 Frank, S.A. (1996) Statistical properties of polymorphism in hostparasite genetics. Evol. Ecol. 10, 307-317

35 Parker, M. (1996) The nature of plant-parasite specificity. Evol. Ecol. $10,319-322$

36 Coustau, C. et al. (2000) Resistance to xenobiotics and parasites: can we count the cost? Trends Ecol. Evol. 15, 378-383

37 Failloux, A-B. et al. (2002) Geographic genetic variation in populations of the dengue virus vector Aedes aegypti. J. Mol. Evol. 55, 653-663

38 Labuda, D. et al. (2000) Archaic lineages in the history of modern humans. Genetics 156, 799-808

39 Armstrong, P.M. and Rico-Hesse, R. (2001) Differential susceptibility of Aedes aegypti to infection by the American and Southeast Asian genotypes of Dengue type 2 virus. Vector Borne Zoonotic Dis. 1, $159-168$

40 Rosen, L. et al. (1983) Transovarial transmission of dengue viruses by mosquitoes: Aedes albopictus and Aedes aegypti. Am. J. Trop. Med. Hyg. 32, 1108-1119

41 Alvarez, M. et al. (2001) Study of biologic attributes of Cuban dengue 2 virus after serial passage in primary dog kidney cells. Int. J. Infect. Dis. 5, 35-39

42 Zeng, L. et al. (1998) Identification of specific nucleotide sequences within the conserved $3^{\prime}$-SL in the dengue type 2 virus genome required for replication. J. Virol. 72, 7510-7522

43 Leitmeyer, K.C. et al. (1999) Dengue virus structural differences that correlates with pathogenesis. J. Virol. 73, 4738-4747

44 Turner, P.E. and Elena, S.F. (2000) Cost of host radiation in an RNA virus. Genetics 156, 1465-1470

45 Novella, I.S. et al. (1999) Lack of evolutionary stasis during alternating replication of an arbovirus in insect and mammalian cells. J. Mol. Biol. 287, 459-465

46 Weaver, S.C. et al. (1999) Genetic and fitness changes accompanying adaptation of an arbovirus to vertebrate and invertebrate cells. J. Virol. 73, 4316-4326

47 Cooper, L.A. and Scott, T.W. (2001) Differential evolution of eastern equine encephalitis virus populations in response to host cell type. Genetics 157, 1403-1412

48 Twiddy, S.S. et al. (2002) Phylogenetic evidence for adaptive evolution of dengue viruses in nature. J. Gen. Virol. 83, 1679-1689

49 Twiddy, S.S. et al. (2003) Inferring the rate and time-scale of dengue viruses evolution. Mol. Biol. Evol. 20, 122-129

50 Murgue, B. et al. (1999) Dengue: an evaluation of dengue severity in French Polynesia based on an analysis of 403 laboratory-confirmed cases. Trop. Med. Int. Health 4, 765-773

51 Ferguson, N.M. et al. (1999) Transmission dynamics and epidemiology of dengue: insights from age-stratified sero-prevalence surveys. Philos. Trans. R. Soc. Lond. B Biol. Sci. 354, 757-768

52 Endy, T.P. et al. (2002) Spatial and temporal circulation of dengue virus serotypes: a prospective study of primary school children in Kamphaeng Phet, Thailand. Am. J. Epidemiol. 156, 52-59

53 Gubler, D.J. (1997) Dengue and dengue hemorrhagic fever: its history and resurgence as a global public health problem. In Dengue and Dengue Hemorrhagic Fever (Gubler, D.J. and Kuno, G., eds), pp. 1-23, CAB International

54 Loroño-Pino, M.A. et al. (1999) Common occurrence of concurrent infections by multiple dengue virus serotypes. Am. J. Trop. Med. Hyg. $51,725-730$

55 Vazeille-Falcoz, M. et al. (1999) Variation in oral susceptibility to dengue type 2 virus of populations of Aedes aegypti from the islands of Tahiti and Moorea, French Polynesia. Am. J. Trop. Med. Hyg. 60, 292-299

56 Tien, T.K. et al. (1999) Aedes aegypti in Ho Chi Minh city (Viet Nam): susceptibility to dengue 2 virus and genetic differentiation. Trans. R. Soc. Trop. Med. Hyg. 93, 581-586

\section{News \& Features on BioMedNet}

Start your day with BioMedNet's own daily science news, features, research update articles and special reports. Every two weeks, enjoy BioMedNet Magazine, which contains free articles from Trends, Current Opinion, Cell and Current Biology. Plus, subscribe to Conference Reporter to get daily reports direct from major life science meetings. 